\title{
CONDICIONES LABORALES Y ESTRES DE LOS TRABAJADORES ADMINISTRATIVOS DE LA MUNICIPALIDAD DISTRITAL DE HUAURA
}

\author{
WORKING CONDITIONS AND STRESS OF THE ADMINISTRATIVE \\ WORKERS OF THE DISTRITAL MUNICIPALITY OF HUAURA
}

Luz Amparo Bernal Villareal ${ }^{1}$, Yuleisi Milagros Yarlaque Roman ${ }^{2}$

\section{RESUMEN}

Objetivo:Identificar la relación existente entre las condiciones laborales y el estrés de los trabajadores administrativos de la Municipalidad Distrital de Huaura. Material y Métodos: El diseño de la investigación fue de corte transversal, tipo correlacional y aplicativo.Resultado: Se obtuvo que las condiciones inadecuadas de trabajo correlacionen directamente con el estrés de los Trabajadores cada vez que se genera inseguridad, ansiedad, frustración to que trae como consecuencia que los trabajadores se estresen dificultando su desenvolvimiento en su quehacer laboral afectando a la Entidad Municipal, a su propia persona y afectando al usuario. Conclusión: El 89\% de los trabajadores indicaron percibir malos condiciones de trabajo y el $85 \%$ presentaron estres severo.

Palabras clave: Condiciones laborales; Estrés; servidores públicos.

\section{ABSTRACT}
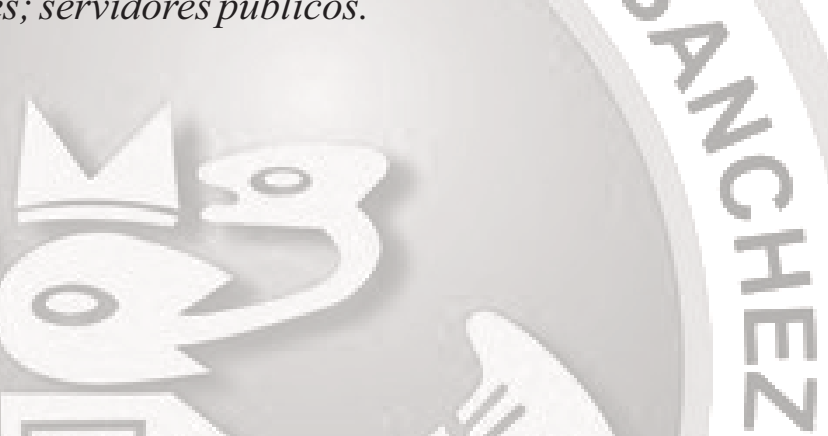

Objective: The identification of the existing relationship between working conditions and the stress of the administrative workers of the District Municipality of Huaura was raised. Material and Methods: The design of the research is of a transversal nature because through the instruments the objectives can be achieved. The type of research used according to the purpose is correlational because it found a relationship between the variables. Likewise, it is applicable since I have taken previous research as reference. Result: It was obtained that the inadequate working conditions correlated directly with the stress of the Workers whenever insecurity, anxiety, frustration is generated, which has as a consequence that the workers are stressed, hindering their development in their work, affecting the Municipal Entity, to his own person and affecting the user. conclusions: the $89 \%$ of the wonker indicated to percive poor working conditions and $85 \%$ perseved severe stress.

Keywords: Working conditions; Stress; public serves.

Asesora en trabajo comunitario y Docente de la Facultad de Ciencias Sociales, Escuela de Trabajo Social. Email.Iuzabernal11@hotmail.com

${ }^{2}$ Lic. Trabajo Social de la Universidad Nacional José Faustino Sánchez Carrion 


\section{INTRODUCCIÓN}

Hoy en día se puede observar en diversas instituciones que las personas asisten a laborar por obligación o porque el dinero no les alcanza y no tienen otra opción, esto genera en ellos estrés y otras consecuencias en contra de su salud, al no gustarle las labores que realizan se obtienen como consecuencias, estrés, mal humor, hasta el punto de hacer las cosas a la deriva y sin ningún cuidado.

En ocasiones el personal se ve sometido a una excesiva carga de trabajo debido a una mala planificación por parte de sus empleadores. Los plazos ajustados y la obligación de terminar tareas elevan los niveles a desarrollar estrés volviendo irritable a la persona y disminuyendo su capacidad para resolver los problemas y ejecutar las tareas previstas.

Al observar el tipo de trato que existe dentro de la Municipalidad Distrital de Huaura se encontró que tanto colaboradores como usuarios tienen un mal trato, siendo este reciproco y generando que las demás personas vean a esta institución como incompetente, sin embargo es importante recalcar que dentro de ello existe un porcentaje menor que si tiene un trato adecuado y es porque se siente satisfecho al realizar sus actividades diarias, a la vez ayuda a que su centro de trabajo se vuelva competente $y$ mejora al crecimiento como institución.

\section{MATERIALES Y MÉTODO}

El diseño de la investigación de corte transversal, porque se utilizaron instrumentos para ambas variables, considerando que están diseñados para aplicarlo tipo encuesta, por lo que se hizo en un solo día, momento y lugar. Así mismo es de tipo correlacional porque hallé relación entre las variables. Población: se tomó como población a los trabajadores administrativos de la municipalidad que hacen un total de 72 personas, teniendo de esta manera 28 del sexo femenino y 44 del sexo masculino que están distribuidos en las 4 sub gerencias siguientes:

Sub gerencia de desarrollo urbano y rural

Sub gerencia de administración y finanzas

Sub gerencia de rentas y desarrollo económico

Sub gerencia de desarrollo humano

\section{Técnicas para el procesamiento de información}

\section{*Análisis Documental}

Mediante el análisis documental y sus respectivos instrumentos, se revisaron fuentes bibliográficas, publicaciones especializadas y portales de internet directamente relacionados con las variables de estudio.

Mediante la técnica el instrumento se pudo identificar como se desarrollaban cada persona dentro de su centro laboral, lo cual nos servirá para desarrollar nuestra investigación y así poder dar respuesta a nuestro objetivo general.

\section{Ficha Técnica de Instrumentos}

\section{*Cuestionario sobre condiciones laborales}

Autora Lic. Azunciona Zelada López, consta de un total de 30 ítems formuladas a manera de preguntas y cuya calificación con una escala de Likert de mayor a menor, es decir: 4-3-2-1.El instrumento de Condiciones Laborales estuvo constituido por 30 ítems.

\section{*Test Valorativo de estrés}

Autora Patricia Curibancoy y Maribel Medina que consta de 20 ítems, los cuales reciben la siguiente calificación: Nunca (1), Algunas veces (2), Frecuentemente (3) Siempre (4).

\section{*Análisis Estadístico}

Se llevó a cabo utilizando el paquete estadístico SPSS 22.0 el cual procesaron los resultados y lograron la interpretación, análisis y discusión de la respuesta al objetivo de la investigación., así mismo contar con las conclusiones, implicando el objetivo que será el producto final de la investigación. 


\section{RESULTADOS}

Tabla 1. Identificar la relación existente entre las condiciones laborales y el estrés de los trabajadores administrativos de la Municipalidad Distrital de Huaura.

\begin{tabular}{lcclcc}
\hline \multicolumn{2}{c}{ CONDICIONES LABORALES } & & \multicolumn{2}{c}{ ESTRES } \\
\hline \multicolumn{2}{l}{ ALTERNATIVAS CANTIDAD $\%$} & ALTERNATIVAS & CANTIDAD & $\%$ \\
\hline BUENAS & 00 & 00 & ESTRÉS & 02 & 03 \\
REGULARES & 08 & 11 & ESTRÉS MODERADO & 09 & 12 \\
MALAS & 64 & 89 & ESTRES SEVERO & 61 & 85 \\
TOTAL & $\mathbf{7 2}$ & $\mathbf{1 0 0}$ & TOTAL & $\mathbf{7 2}$ & $\mathbf{1 0 0}$ \\
\hline
\end{tabular}

De 72 trabajadores que representa el $100 \%$ de la población evaluada, según el test de condiciones de trabajo, ninguno que es el $0 \%$ indico buenas condiciones, 08 que representa el $11 \%$ indico regulares condiciones; por lo que 64 trabajadores que representa el $89 \%$ indicaron percibir malas condiciones de trabajo, ubicándose en el rango negativo, Asimismo de 72 trabajadores evaluados que representa el $100 \%$ de la población; 02 que representa el $03 \%$ presenta estrés leve; 09 que representa el $12 \%$ presenta estrés moderado; por lo que 61 personas que representa el $85 \%$ presentan un estrés severo. Ubicándose también en un rango negativo.

Correlación entre las variables de estudio: Condiciones de trabajo y estrés de los trabajadores administrativos

Correlaciones bi variadas de Rango de Spearman de la población total $=72$

\begin{tabular}{|c|c|c|c|c|}
\hline \multirow{8}{*}{$\begin{array}{l}\text { Rho de } \\
\text { Spearman }\end{array}$} & \multirow{5}{*}{$\begin{array}{l}\text { Condiciones } \\
\text { Laborales }\end{array}$} & \multirow{4}{*}{$\begin{array}{l}\text { Coeficiente de correlación } \\
\text { Sig. (bilateral) }\end{array}$} & \multirow{2}{*}{$\begin{array}{l}\text { Condiciones } \\
\text { Laborales }\end{array}$} & \multirow{3}{*}{$\begin{array}{l}\text { Nivel de } \\
\text { Estrés } \\
, 539 * *\end{array}$} \\
\hline & & & & \\
\hline & & & 1,000 & \\
\hline & & & & 000 \\
\hline & & $\mathrm{N}$ & 72 & 72 \\
\hline & $\begin{array}{l}\text { Nivel de } \\
\text { Estrés }\end{array}$ & Coeficiente de correlación &, $539 * *$ & 1,000 \\
\hline & & Sig. (bilateral) &, 000 & \\
\hline & & $\mathrm{N}$ & 72 & 72 \\
\hline
\end{tabular}

** La correlación es significativa al nivel 0,001 (bilateral)

\section{Correlación entre condiciones laborales y nivel de estrés}

En el cuadro referente a la correlación negativa entre las variables de motivación y rendimiento académico se encuentra una buena correlación de 0.539, el coeficiente es significativo, porque encontramos una s. 0.000 al nivel de 0.085 de error de confianza. En tal correlación de Sperman nos indica la dirección de las dos variables de investigación, por lo tanto, podemos rechazar la hipótesis nula y aceptar la hipótesis alterna (se acepta la hipótesis alterna porque la sig. es menor que el error de confianza). Señalando que las Condiciones Laborales se relacionan con el Nivel de Estrés en los trabajadores de administración de la Municipalidad del Distrito de Huaura.

\section{DISCUSIÓN}

El resultado se apoya en la teoría transaccional de (Lazarus, 2010) que sostiene que el estrés se presenta debido a que la situación o contexto, que es percibida por la persona como amenazante, la misma que agrava afectando los recursos propios y capacidad de afronte de la persona, poniendo en peligro su bienestar personal.

Por otro lado, cual menciona que el estrés laboral es uno de los temas que actualmente más se está investigando ya que puede afectar a la actividad y rendimiento en el trabajo de cualquier ser humano, $\mathrm{y}$ también a la vida personal.

Considerando que estudiosos como Lazarus y Folkman (1986) estudiosos del estrés, autores de la Teoría Transaccional afirmaron que, el estrés surge del modo como la persona percibe e interpreta los acontecimientos que ocurren en el ambiente exterior. Seguidamente sostienen que, esta percepción depende del contexto en que la persona se desenvuelve. Asimismo, (Selye, 1975) señala con mucha claridad que el estrés "perjudicante" o "desagradable" se produce por una carga de trabajo no asimilable debido a las condiciones inadecuadas en las que se desarrollan que provocan un desequilibrio fisiológico y psicológico, que termina en una reducción de la productividad del individuo, la aparición de enfermedades psicosomáticas y el envejecimiento acelerado.

Por otro lado en investigaciones específicas; en su investigación titulada: Estrés laboral y calidad del cuidado del enfermero del Minsa; Lima - Perú; concluyeron de que el $96 \%$ presentaban un estrés laboral medio y alto y que esto influía en la mala calidad del cuidado que ejercían estos como trabajadores del sector salud. 
(Huamán y Menacho, 2007) en su investigación; Estrés y desempeño laboral de los enfermeros hospitalarios de Huaraz; Ancash - Perú. Concluyeron que el $87 \%$ de los evaluados tenían un desempeño no satisfactorio porque el $63 \%$ de los evaluados padecían de un estrés laboral muy alto.

Asimismo, Vasquez y Chugo(2003), en su investigación: Fuentes del estrés laboral en profesores de la Institución Educativa Estatal del distrito de Trujillo; La Libertad - Perú, concluyeron con el $75 \%$ padecía de un estrés muy alto; medidos con una escala que denominaron Escala FELP, lo que a criterios de dichos estudiosos fueron causados por el tipo de condiciones de trabajo, la infraestructura donde laboraban, la falta de empatía de los alumnos y el tipo de gestión educativa por parte de los directivos de las Instituciones Educativas Estatales.

Teniendo estos resultados como antecedentes considero que los resultados encontrados en mi investigación, tiene sustento que le da solidez a lo encontrado: De 72 trabajadores que representa el $100 \%$ de la población evaluada, según el Test de Condiciones de Trabajo, ninguno que es el $0 \%$ indico buenas condiciones, 08 que representa el $11 \%$ indico regulares condiciones; por lo que 64 trabajadores que representa el $89 \%$ indicaron percibir malas condiciones de trabajo, ubicándose en el rango negativo. Asimismo, de 72 trabajadores evaluados que representa el $100 \%$ de la población evaluada según êl Test Valorativo del Estrés; 02 que representa el $03 \%$ presenta estrés leve; 09 que representa el $12 \%$ presenta estrés moderado; por lo que 61 personas que representa el $85 \%$ presentan un estrés severo. Los trabajadores de la Municipalidad Distrital de Huaura padecen de un estrés severo, que es un problema serio de salud pública y que requiere alternativas de atención al respecto; en caso contrario este problema tendera a complicarse más aún, complicando la situación personal de los mismos, se sus familias y de la propia Institución.

Finalmente quiero señalar que (Jara, 2012) en su investigación Condiciones de Trabajo y Estrés de los Operarios de la Planta de cerdos Santa Rosa de Chincha de la Empresa Dan Fernando S.A.; Ica Perú.-Encontró que de 25 trabajadores que representa el $100 \%$ todos percibían unas buenas y regulares condiciones de trabajo. Asimismo, señala que los 25 trabajadores es decir el 100\% de los evaluados ninguno presentaba un estrés severo (dañino). Señala que esta evaluación la desarrollo luego de implementar una serie de Programas y acciones de atención personalizada y familiar a los operarios en dicha empresa. Contrariamente a esta posibilidad las buenas condiciones de trabajo evitan la generación del estrés laboral.

Por lo tanto, aceptar la hipótesis alterna de investigación y rechazar la hipótesis nula presentada, demuestra que existe una buena relación directa negativa entre las dos variables por lo que existe relación entre las variables: condiciones laborales y el nivel de estrés de los trabajadores administrativos de la Municipalidad Distrital de Huaura . Esta decisión se sustenta en el valor de significancia de 0.000 a un nivel de error de confianza de 0.05 hallado en la correlación de rango de Spearman.

\section{CONCLUSIONES}

De 72 trabajadores que representa el $100 \%$ de la población evaluada, según el test de condiciones de trabajo, ninguno que es el $0 \%$ indico buenas condiciones, 08 que representa el $11 \%$ indico regulares condiciones; por lo que 64 trabajadores que representa el $89 \%$ indicaron percibir malas condiciones de trabajo, ubicándose en el rango negativo, Asimismo de 72 trabajadores evaluados que representa el $100 \%$ de la población; 02 que representa el $03 \%$ presenta estrés leve; 09 que representa el $12 \%$ presenta estrés moderado; por lo que 61 personas que representa el $85 \%$ presentan un estrés severo. Ubicándose también en un rango negativo.

El resultado hallado entre las variables condiciones laborales y rendimiento académico se encuentra una buena correlación de 0.539 , el coeficiente es significativo, porque encontramos una s. 0.000 al nivel de 0.085 de error de confianza. 


\section{RECOMENDACIONES}

1.- Es recomendable que en las instituciones como la Municipalidad Distrital de Huaura, la autoridades políticas desde el Alcalde y las Gerencias respectivas que manejan la dinámica organizacional, asuman medidas correctivas que repercuta positivamente en el mejoramiento de las condiciones laborales a fin de que estas se vean mejoradas y que los trabajadores disfruten de esas posibilidades de manera consciente el mismo que permitirá un compromiso en ellos desempeñándose con más eficiencia y con satisfacción al ver mejorados el contexto donde se desenvuelven diariamente.

2.- Es recomendable también diseñar algunas estrategias que contribuyan a la mejora de las condiciones personales del trabajador, así por ejemplo deberán planificarse talleres de capacitación en habilidades sociales, en técnicas de relajación; en gerencia y atención al público, en competencias relacionadas a la tecnología moderna que permanentemente va cambiando y evolucionando y de este modo los trabajadores no se sientan postergados en su quehacer cotidiano en sus respectivos puestos de trabajo.

3.- La universidad como institución rectora de la educación, la cultura y la capacitación puede y debería ofrecer a las instituciones de su área de influencia, convenios y acuerdos que le permitan de manera comprometida desarrollar periódicamente planes y proyectos de capacitación en beneficio del personal que labora en las diferentes entidades como la Municipalidad Distrital de Huaura y de este modo ser útil en la mejora de la gestión de recursos humanos, así como también en el manejo presupuestario y mejoramiento de los servicios de estas entidades importantes.

\section{AGRADECIMIENTO}

A los trabajadores administrativos de la municipalidad distrital de Huaura por brindarnos la información necesaria para realizar la investigación científica y así mismo al equipo de investigación.

\section{REFERENCIAS BIBLIOGRÁFICAS}

Huaman, R.NM \& Menacho V.B.A. (2007). Estres y desempeño laboral de las enfermeras(os) hospitalarios, hhuaraz.tesis de licenciatura. Universidad los Angeles de Chimbote.

Jara Violeta (2012). En su investigación Condiciones de Trabajo y Estrés de los Operarios de la Planta de cerdos Santa Rosa de Chincha de la Empresa Dan Fernando S.A.; Ica-Perú.

Lazarus,R.\&. Folkman,S.(1986). Estres y proceso cognitivos: el concepto de afrontamiento. 1984-1986, Barcelona. Edicion Martinez roca.p. 141

Lazarus Richard (2000); Estrés y Emociones: El manejo e implicancias en nuêstra salud. Ed. Descle Bruwer. E.U.A.

Selye Hans (1975). Tensión sin angustia; Editorial Gualrdarrama, Madrid-España

Vasquez \& chugo (2003). Fuentes del estres laboral en profesores de la I.E. estatal del distrito de Trujillo.Peru.

\section{HUACHO}

